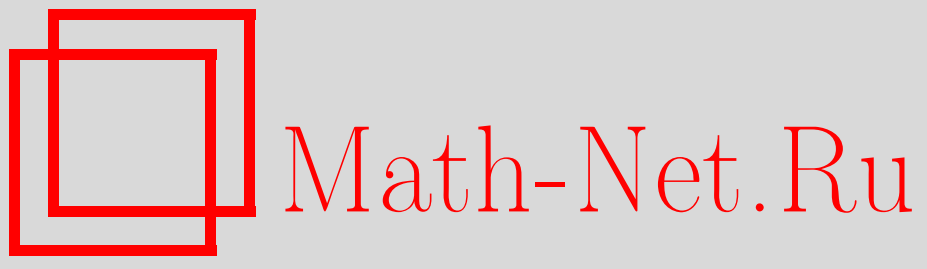

И. А. Дынников, Трехстраничный подход в теории узлов. Универсальная полугруппа, Функи. анализ и его прил., 2000, том 34, выпуск 1, 29-40

DOI: https://doi.org/10.4213/faa280

Использование Общероссийского математического портала MathNet.Ru подразумевает, что вы прочитали и согласны с пользовательским соглашением

http://www . mathnet.ru/rus/agreement

Параметры загрузки:

IP : 3.81 .55 .215

26 апреля 2023 г., 06:42:04

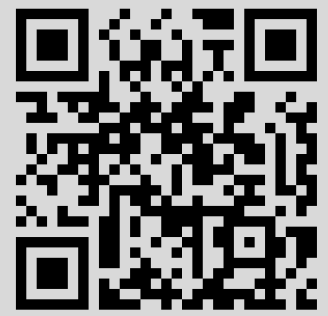


Функциональный анализ и его приложения

2000, т. 34, вып. 1, с. 29-40

УДК 515.164.63

\title{
Трехстраничный подход в теории узлов. Универсальная полугруппа*
}

\author{
(c) 2000. И. А. Дынников
}

В предыдущей работе автора [9] указывается способ кодирования неориентированных зацеплений, вложенных в трехстраничную книгу, словами в 12-буквенном алфавите и приводится конечный набор локальных замен, реализующих эквивалентность зацеплений. Здесь мы дадим простую алгебраическую интерпретацию этих результатов, а именно укажем конечно представленную полугруппу, центр которой классифицирует неориентированные зацепления. Мы докажем, что группа кос $B_{\infty}$ на бесконечном числе нитей реализуется как коммутант группы, порожденной двумя образуюшими и тремя соотношениями

$$
\left[[a, b], a^{2} b a^{-2}\right]=\left[[a, b], b^{2} a b^{-2}\right]=\left[[a, b],\left[a^{-1}, b^{-1}\right]\right]=1,
$$

где $[x, y]=x y x^{-1} y^{-1}$. Мы также обобщим конструкцию нашей полугруппы на случай оснащенных зацеплений.

Основные результаты настоящей работы анонсированы в [4]. Некоторые другие результаты, в частности о частичном алгоритме распознавания тривиального узла с помощью трехстраничных диаграмм, см. в [5].

\section{§1. Трехстраничные плетения и косы}

В настоящей работе мы будем использовать определения и обозначения из работы [9]. Напомним, что в [9, определение 1] мы ввели понятие трехстраничной диаграммы зацепления как зацепления, вложенного в трехстраничную книгу $\mathbb{Y}$ (т. е. объединение трех полуплоскостей $P_{1}, P_{2}, P_{3}$, прикрепленных к общей граничной прямой $l$ ) и удовлетворяющего условиям трансверсальности и монотонности. Такая трехстраничная диаграмма $L$ кодируется полностью сбалансированным словом $w_{L}$ в алфавите $\mathbb{A}=\left\{a_{i}, b_{i}, c_{i}, d_{i}\right\}_{i \in \mathbb{Z}_{3}}$ (см. $\left.[9, \S 2]\right)$. Теорема 2 в [9] утверждает, что два полностью сбалансированных слова в алфавите $\mathbb{A}$ задают эквивалентные зацепления в $\mathbb{R}^{3}$ тогда и только тогда, когда одно можно получить из другого конечной последовательностью локальных замен из следующего списка:

$$
\left.\begin{array}{rl}
a_{i+1} d_{i-1} & \leftrightarrow a_{i} \leftrightarrow a_{i-1} b_{i+1}, \\
d_{i+1} d_{i-1} & \leftrightarrow b_{i} \leftrightarrow a_{i-1} c_{i+1}, \\
d_{i+1} c_{i-1} & \leftrightarrow c_{i} \leftrightarrow b_{i-1} c_{i+1}, \\
a_{i+1} c_{i-1} & \leftrightarrow d_{i} \leftrightarrow b_{i-1} b_{i+1},
\end{array}\right\}
$$

* Работа выполнена при частичной поддержке Российского фонда фундаментальных исследований (грант № 99-01-00090). 


$$
\left.\begin{array}{rl}
a_{i-1} b_{i-1} w_{i} & \leftrightarrow w_{i} a_{i-1} b_{i-1}, \\
d_{i-1} c_{i-1} w_{i} & \leftrightarrow w_{i} d_{i-1} c_{i-1}, \\
d_{i-1} b_{i-1} w_{i} & \leftrightarrow w_{i} d_{i-1} b_{i-1},
\end{array}\right\}
$$

где $i \in \mathbb{Z}_{3}, w_{i} \in X_{i}, X_{i}=\left\{a_{i}, b_{i}, c_{i}, d_{i}, b_{i-1} a_{i} d_{i-1}, b_{i-1} b_{i} d_{i-1}, b_{i-1} c_{i} d_{i-1}, b_{i-1} d_{i} d_{i-1}\right\}$.

Полугруппа, получающаяся факторизацией полугруппы $W_{0}$ полностью сбалансированных слов по соотношениям (2)-(4), коммутативна и свободно порождена изотопическими классами неразводимых зацеплений. Умножение в этой полугруппе отвечает взятию объединения зацеплений, разделенных некоторой сферой. Ниже мы построим конечно представленную полугруппу, в которой указанная полугруппа зацеплений является центром.

Пусть $Y$ - полугруппа с множеством образующих $\mathbb{A}$ и следующими 55 соотношениями:

$$
\begin{gathered}
a_{i}=a_{i+1} d_{i-1}, \quad b_{i}=a_{i-1} c_{i+1}, \quad c_{i}=b_{i-1} c_{i+1}, \quad d_{i}=a_{i+1} c_{i-1}, \\
d_{1} d_{2} d_{3}=1, \\
b_{i} d_{i}=d_{i} b_{i}=1, \\
v w=w v,
\end{gathered}
$$

где $v \in\left\{a_{i-1} b_{i-1}, d_{i-1} c_{i-1}, b_{i+1} d_{i-1} d_{i+1} b_{i-1}\right\}, w \in\left\{a_{i}, b_{i}, c_{i}, b_{i-1} d_{i} d_{i-1}\right\}, i \in \mathbb{Z}_{3}$.

Теорема 1. 1) Две трехстраничные диаграммь $L_{1} u L_{2}$ представляют изотопнье неориентированнье зачепления в $\mathbb{R}^{3}$ тогда и только тогда, когда в полугруппе $Y$ имеет место равенство $w_{L_{1}}=w_{L_{2}}$.

2) Элемент $y \in Y$ можно записать словом вида $w_{L}$, где $L$ - некоторая трехстраничная диаграмма зачепления, тогда и только тогда, когда у иентральный элемент.

Таким образом, центр полугруппы $Y$ классифицирует все неориентированные зацепления.

ДокАЗАТЕЛьСтво. Сначала мы обобщим понятие трехстраничной диаграммы зацепления.

Пусть П - некоторая плоскость, перпендикулярная линии переплета $l, r_{1}$, $r_{2}, r_{3}$ - три луча: $r_{i}=\Pi \cap P_{i}$, и $O$ - точка пересечения плоскости $\Pi$ с линией переплета, $O=\Pi \cap l$. На каждом луче $r_{i}$ отметим бесконечное число равноудаленных точек:

$$
E_{i, k} \in r_{i}, \quad \overrightarrow{O E}_{i, 1}={\overrightarrow{E_{i, k} E_{i, k+1}}}_{i}, \quad \text { где } i \in \mathbb{Z}_{3}, k \in \mathbb{N} .
$$

Обозначим через $E$ объединение этих трех серий точек, $E=\left\{E_{i, k}\right\}, i \in \mathbb{Z}_{3}$, $k \in \mathbb{N}$.

Используя ортогональные проекции, представим евклидово пространство $\mathbb{R}^{3}$ как прямое произведение $\Pi \times l$ и отождествим $l$ с вещественной прямой $\mathbb{R}$. Обозначим множества $E \times 0$ и $E \times 1$ через $E^{(0)}=\left\{E_{i, k}^{(0)}\right\}$ и $E^{(1)}=\left\{E_{i, k}^{(1)}\right\}$ соответственно. Плоскости $\Pi \times 0$ и $\Pi \times 1$ обозначим через $\Pi^{(0)}$ и $\Pi^{(1)}$.

ОПРЕДЕЛЕНИЕ 1. Трехстраничным плетением назовем ломаную линию $T \subset$ $\mathbb{Y} \cap(\Pi \times[0,1])$, такую, что

1) $T \cap\left(\Pi^{(0)} \cup \Pi^{(1)}\right)=\partial T=E^{(0)} \cup E^{(1)}$;

2) ломаная $T$ трансверсальна линии переплета $l$ (см. [9, определение 1]); 
3) $T$ удовлетворяет условию монотонности из $[9$, определение 1$]$.

Два трехстраничных плетения называются эквивалентныли, если они (объемлемо) изотопны в $\Pi$ × $[0,1]$ (граница $\partial T$ предполагается фиксированной при изотопии).

При этом под ломаной линией мы подразумеваем одномерное PL-подмногообразие в $\mathbb{R}^{3}$, каждая компонента которого компактна. Пример трехстраничного плетения показан на рис. 1.

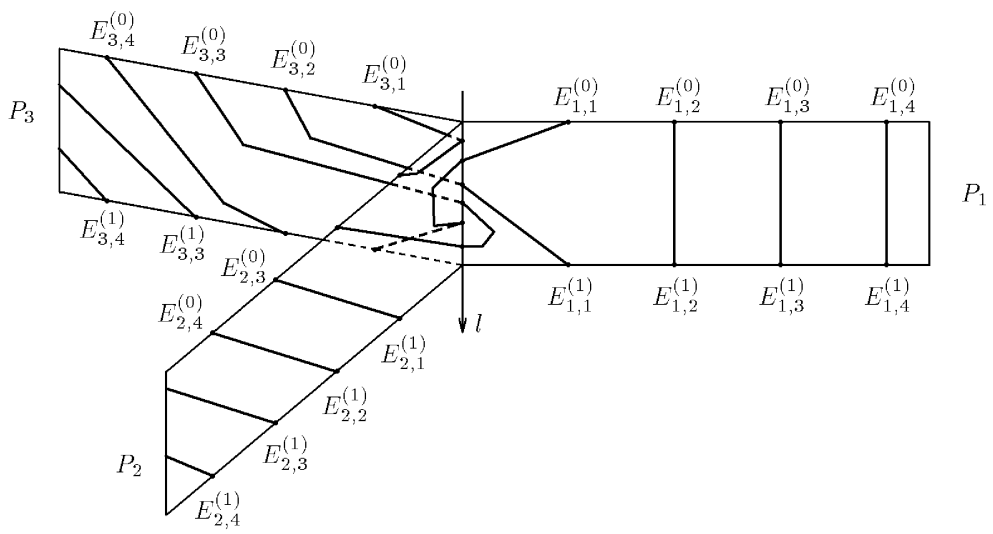

Рис. 1. Трехстраничное плетение

Композиция трехстраничных плетений определяется по аналогии с композицией обычных плетений и кос. Пусть $\tau_{1}, \tau_{2}$ - следующие аффинные преобразования пространства $\mathbb{R}^{3}$ :

$$
\tau_{1}(Q, t)=(Q, t / 2), \quad \tau_{2}(Q, t)=(Q,(t+1) / 2), \quad Q \in \Pi, t \in \mathbb{R} .
$$

Композииией $T_{1} \circ T_{2}$ трехстраничных плетений $T_{1}$ и $T_{2}$ называется объединение

$$
\tau_{1}\left(T_{1}\right) \cup \tau_{2}\left(T_{2}\right)
$$

Эта операция корректно определена на классах эквивалентности трехстраничных плетений и задает на множестве этих классов структуру полугруппы с единицей $I=E \times[0,1]$. Мы утверждаем, что эта полугруппа есть в точности $Y$.

Действительно, так же, как и в случае трехстраничных диаграмм зацеплений, любое трехстраничное плетение $T$ кодируется словом $w_{T}$ в алфавите $\mathbb{A}$. Для трехстраничных плетений имеет место аналог второго утверждения из предложения 1 в [9]: трехстраничные плетения $T$ и $T^{\prime}$ объемлемо изотопны в $\mathbb{Y} \cap(\Pi \times[0,1])$ тогда и только тогда, когда слова $w_{T}$ и $w_{T^{\prime}}$ совпадают. Образующие $a_{i}, b_{i}, c_{i}, d_{i}$ полугруппы трехстраничных плетений показаны на рис. 2. Часть этих трехстраничных плетений, не показанная на рисунке, имеет вид $E_{i} \times[0,1]$, где $E_{i}=\left\{E_{i, k}\right\}_{k \in \mathbb{N}}$.

Нарисовав соответствующие картинки, легко проверить, что соотношения (5)-(8) выполнены в полугруппе трехстраничных плетений. Мы должны убедиться, что этих соотношений достаточно для реализации эквивалентности трехстраничных плетений. Кроме того, мы утверждаем, что любая ломаная линия $T \subset \Pi \times[0,1]$ с границей $\partial T=E^{(0)} \cup E^{(1)}$, такая, что при некоторых 
$n_{1}, n_{2}, n_{3} \in \mathbb{Z}$ и всех достаточно больших $k$ отрезки $\left[E_{i, k}^{(0)}, E_{i, k+n_{i}}^{(1)}\right]$ содержатся в $T$, изотопна в $\Pi \times[0,1]$ некоторому трехстраничному плетению.

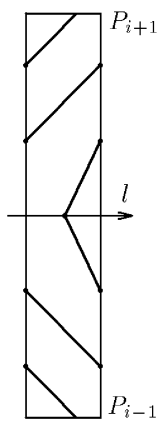

$a_{i}$

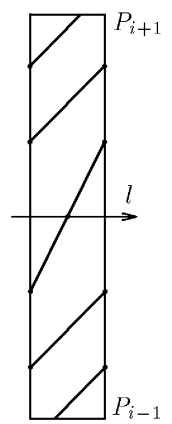

$b_{i}$

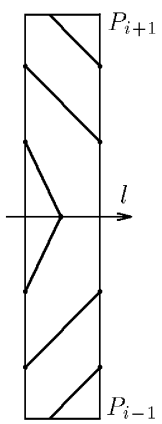

$c_{i}$

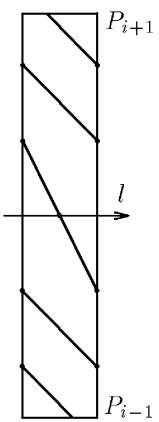

$d_{i}$

Рис. 2. Геометрическая реализация образующих полугруппы $Y$

Доказательство этих фактов по существу повторяет доказательство теоремы 2 из [9]. Мы прокомментируем только некоторые отличия соотношений (5)-(8) от (2)-(4).

Прежде всего, в полугруппе трехстраничных зацеплений мы не писали соотношений $b_{i} d_{i}=d_{i} b_{i}=1$, поскольку слова $b_{i} d_{i}$ и $d_{i} b_{i}$ несбалансированы. Очевидно, что в полугруппе трехстраничных плетений элементы $b_{i}$ и $d_{i}$ взаимно обратны. Следовательно, соотношения $a_{i}=a_{i+1} d_{i-1}$ и $c_{i}=b_{i-1} c_{i+1}$ из (5) теперь эквивалентны соотношениям $a_{i}=a_{i-1} b_{i+1}$ и $c_{i}=d_{i+1} c_{i-1}$ соответственно. А соотношения $b_{i}=d_{i+1} d_{i-1}$ и $d_{i}=b_{i-1} b_{i+1}$ эквивалентны $(6)$.

Наконец, по сравнению с (3)-(4) в списке (8) мы опустили соотношения вида $v w=w v$, где слово $v$ то же, что и в $(8)$, а слово $w$ - одно из следующих:

$$
b_{i-1} a_{i} d_{i-1}, \quad b_{i-1} b_{i} d_{i-1}, \quad b_{i-1} c_{i} d_{i-1} .
$$

В полугруппе $Y$ мы имеем

$$
\begin{aligned}
b_{i-1} a_{i} d_{i-1} & =b_{i-1} a_{i+1} d_{i-1} d_{i-1}=b_{i-1} a_{i+1} b_{i+1} b_{i} d_{i-1}=a_{i+1} b_{i+1} b_{i-1} b_{i} d_{i-1} \\
& =a_{i+1} d_{i-1} d_{i} b_{i-1} b_{i} d_{i-1}=a_{i} d_{i} b_{i-1} b_{i} d_{i-1} \\
b_{i-1} b_{i} d_{i-1} & =\left(b_{i-1} d_{i} d_{i-1}\right)^{-1}, \\
b_{i-1} c_{i} d_{i-1} & =b_{i-1} b_{i-1} c_{i+1} d_{i-1}=b_{i-1} d_{i} d_{i+1} c_{i+1} d_{i-1}=b_{i-1} d_{i} d_{i-1} d_{i+1} c_{i+1} \\
& =b_{i-1} d_{i} d_{i-1} b_{i} b_{i-1} c_{i+1}=b_{i-1} d_{i} d_{i-1} b_{i} c_{i} .
\end{aligned}
$$

Таким образом, если элемент $v \in Y$ коммутирует с $a_{i}, b_{i}, c_{i}, d_{i}, b_{i-1} d_{i} d_{i-1}$, то он коммутирует и с элементами (9).

Далее мы будем отождествлять полугруппу $Y$ с полугруппой изотопических классов трехстраничных плетений. Для завершения доказательства теоремы 1 мы должны еще описать центр полугруппы $Y$. Нам понадобится следующее определение.

ОПредЕлЕниЕ 2. Скажем, что трехстраничное плетение $T$ отделимо, если найдется трехмерный шар $\mathcal{B} \subset \Pi \times[0,1]$, такой, что

1) его граница $\partial \mathcal{B} \cong S^{2}$ не пересекает $T$, и, таким образом, пересечение $T \cap \mathcal{B}$ состоит только из замкнутых компонент плетения $T$; 
2) оставшаяся часть $T-(T \cap \mathcal{B})$ представляет собой трехстраничное плетение, эквивалентное единичному плетению $I=E \times[0,1]$.

Очевидно, что трехстраничное плетение, эквивалентное некоторому отделимому, также отделимо. Таким образом, можно говорить об отделимых элементах полугруппы $Y$. Каждое отделимое трехстраничное плетение $T$ задает зацепление в $\mathbb{R}^{3}$, которое представляет собой пересечение соответствующего шара $\mathcal{B}$ с $T$. Следующие два утверждения очевидны.

ЛЕмма 1. Элемент полугруппь $Y$ отделим тогда и только тогда, когда он представим полностью сбалансированным словом в алфавите $\mathbb{A}$.

Лемма 2. Пусть $w$ - произвольное полностью сбалансированное слово. Тогда элемент $y \in Y$ отделим в том и только том случае, когда отделим элемент $y w \in Y$.

Следуюшая лемма завершает доказательство теоремы 1.

ЛЕмма 3. Элемент полугруппь $Y$ отделим тогда и только тогда, когда он центральньй.

ДокАЗАТЕльство. Нетривиальной является только часть «тогда». Пусть трехстраничное плетение $T$ представляет элемент $y \in Y$ и $w_{T}=x_{1} \ldots x_{m}$ соответствуюшее слово, $x_{k} \in \mathbb{A}, k=1, \ldots, m$. Из геометрической реализации соотношения $b_{i}^{N} a_{i} d_{i}^{N} y=y b_{i}^{N} a_{i} d_{i}^{N}$ легко вывести, что $x_{1}+\cdots+x_{m}=0 \in \mathbb{Z}^{3}$.

Отсюда следует, что для достаточно большого $N$ слово $a_{1}^{N} a_{2}^{N} w_{T} c_{2}^{N} c_{1}^{N}$ сбалансировано. Но $a_{1}^{N} a_{2}^{N} w_{T} c_{2}^{N} c_{1}^{N}=w_{T} a_{1}^{N} a_{2}^{N} c_{2}^{N} c_{1}^{N}$ и слово $a_{1}^{N} a_{2}^{N} c_{2}^{N} c_{1}^{N}$ сбалансировано. Из леммы 2 следует, что элемент $y=w_{T}$ отделим.

Теперь мы перейдем к рассмотрению косоподобных элементов полугруппы $Y$.

ТЕОрема 2. Пусть $D$ - еруппа, порожденная образуюшими $d_{1}, d_{2}, d_{3} u$ следуюшими семью соотношениями:

$$
\begin{aligned}
d_{1} d_{2} d_{3} & =1, \\
d_{i} u_{i} & =u_{i} d_{i}, \\
u_{i} u_{i+1} & =u_{i+1} u_{i},
\end{aligned}
$$

где $u_{i}=d_{i+1}^{-1} d_{i-1} d_{i+1} d_{i-1}^{-1}, i \in \mathbb{Z}_{3}$. Пусть $D_{i}-$ noдарynna арynnь $D$, nopoжденная элементами $d_{i}, u_{i+1}, u \widehat{D}_{i}$ - пересечение $D_{i+1} \cap D_{i-1}, i \in \mathbb{Z}_{3}$. Тогда

1) $D$ изоморфна аруппе обратимых элементов полугруппь $Y$, причем изоморфизм задается отождествлением образуюших $d_{i}$ группь $D$ c соответствующими образующими полугруппьи $Y$;

2) коммутант $D^{\prime}=\left\{x y x^{-1} y^{-1} \mid x, y \in D\right\}$ изоморфен группе кос $B_{\infty} н$ бесконечном числе нитей, и мь имеем $D / D^{\prime}=\mathbb{Z}^{2}$;

3) подаруппь $D_{i}$ и $\widehat{D}_{i}$ являются иентрализаторами друг друга для любого $i \in \mathbb{Z}_{3}$;

4) nодаруппа $\widehat{D}_{i}$ изоморфна аруппе кос $B_{\infty}$ при всех $i \in \mathbb{Z}_{3}$;

5) подаруппа $D_{i}$ изоморфна полупрямому произведению $B \backslash \mathbb{Z}$ при всех $i \in \mathbb{Z}_{3}$. 
ЗАМЕЧАНИЕ 1 . В предположении, что $d_{3}=d_{2}^{-1} d_{1}^{-1}$, соотношения (11) и (12) равносильны следующим:

$$
\begin{gathered}
d_{1} d_{3} d_{1}^{-1} d_{2} d_{1}=d_{2} d_{1} d_{2}^{-1} d_{3} d_{2}=d_{3} d_{2} d_{3}^{-1} d_{1} d_{3} \\
u_{3} u_{2}=u_{2} u_{3} .
\end{gathered}
$$

Таким образом, число образующих и соотношений, задающих группу $D$, можно уменьшить до двух и трех соответственно. Другая запись соотношений (13) и (14) дана в (1), где мы положили $a=d_{1}^{-1}, b=d_{2}$.

ДокАЗАТЕльство. Для начала мы напомним некоторые общеизвестные факты о группах кос. Пусть $F$ - произвольное локально конечное подмножество плоскости П и $B_{F}$ - группа, элементами которой являются изотопические классы кос, т.е. ломаных линий $T \subset \Pi \times[0,1]$, таких, что

1) $\partial T=(F \times\{0\}) \cup(F \times\{1\})$;

$2)$ ограничение ортогональной проекции $\Pi \times[0,1] \rightarrow[0,1]$ на каждую связную компоненту (нить) косы $T$ есть монотонная функция;

3) все, кроме конечного числа, нити косы $T$ представляют собой прямолинейные отрезки, параллельные линии $l$.

Если имеется биекция между двумя локально конечными подмножествами $F_{1}, F_{2} \subset \Pi$, то группы $B_{F_{1}}$ и $B_{F_{2}}$ изоморфны, причем изоморфизм определяется любым (кусочно-линейным) гомеоморфизмом $h: \Pi \rightarrow \Pi$, таким, что $h\left(F_{1}\right)=F_{2}$. В частности, если $F-$ множество из $n$ точек, то группа $B_{F}$ изоморфна apтиновской группе кос $B_{n}$ на $n$ нитях. Если $F$ - бесконечное множество, то группа $B_{F}$ изоморфна группе кос $B_{\infty}$ на бесконечном числе нитей, которая является прямым пределом последовательности $B_{1} \subset B_{2} \subset B_{3} \subset \ldots$.

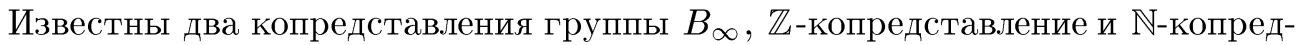
ставление. Обе группы $B_{\mathbb{Z}}$ и $B_{\mathbb{N}}$ порождены образующими $\sigma_{k}$, где $k \in \mathbb{Z}$ для группы $B_{\mathbb{Z}}$ и $k \in \mathbb{N}$ для группы $B_{\mathbb{N}}$, и следующими соотношениями:

$$
\begin{gathered}
\sigma_{j} \sigma_{k}=\sigma_{k} \sigma_{j}, \quad k>j+1, \\
\sigma_{k} \sigma_{k+1} \sigma_{k}=\sigma_{k+1} \sigma_{k} \sigma_{k+1},
\end{gathered}
$$

где $k, j \in \mathbb{Z}$ или $\mathbb{N}$ соответственно.

Пусть $H$ - группа обратимых элементов полугруппы $Y$. Трехстраничное плетение $T$ представляет обратимый элемент из $Y$, если и только если существует сохраняющая границу $\partial T$ изотопия от $T$ к некоторой ломаной линии $T^{\prime} \subset \Pi \times[0,1]$, такой, что

1) ограничение проекции $\Pi \times[0,1] \rightarrow[0,1]$ на каждую связную компоненту ломаной $T^{\prime}$ есть монотонная функция;

2) для некоторых $n_{1}, n_{2}, n_{3} \in \mathbb{Z}$ в плетении $T^{\prime}$ содержится прямолинейный отрезок $\left[E_{i, k}^{(0)}, E_{i, k+n_{i}}^{(1)}\right]$ при всех $i \in \mathbb{Z}_{3}$ и достаточно большом $k$.

Напомним (см. $[9, \S 2])$, что алфавит $\mathbb{A}$ отождествлен с некоторым подмножеством целочисленных векторов в $\mathbb{R}^{3}$. Это позволяет определить отображение $\varphi$ из множества $W$ всех слов в $\mathbb{Z}^{3}$ по формуле $\varphi\left(x_{1} \ldots x_{m}\right)=x_{1}+\cdots+x_{m}$, где $x_{k} \in \mathbb{A}$. Легко видеть, что это отображение факторизуется до гомоморфизма $Y \rightarrow \mathbb{Z}^{3}$, который мы также обозначим через $\varphi$. Упомянутая выше тройка $\left(n_{1}, n_{2}, n_{3}\right)$ целых чисел - это в точности $\varphi\left(w_{T}\right)$. Образ $\varphi(H)$ изоморфен группе $\mathbb{Z}^{2}$ и состоит из векторов $\left(n_{1}, n_{2}, n_{3}\right) \in \mathbb{Z}^{3}$ с нулевой суммой координат: 
$n_{1}+n_{2}+n_{3}=0$. Ядро гомоморфизма $\operatorname{ker}\left(\left.\varphi\right|_{H}\right)$ по построению изоморфно группе кос $B_{E} \cong B_{\infty}$, и мы имеем $H / B_{E}=\mathbb{Z}^{2}$.

Мы собираемся доказать, что $D \cong H$. Соотношения (10)-(12) выполнены в группе $H$ (в действительности это в точности те из соотношений (5)-(8), которые содержат только образующие $\left.b_{i}, d_{i}\right)$. Таким образом, имеется гомоморфизм $\eta: D \rightarrow H$, переводящий образующие $d_{i}$ группы $D$ в соответствующие элементы $d_{i}$ группы $H$.

Из соотношений (10)-(12) тривиальным образом следует, что

$$
D / D^{\prime} \cong \mathbb{Z}^{2} \quad \text { и } \quad \eta\left(D^{\prime}\right) \subset B_{E} .
$$

Таким образом, достаточно показать, что ограничение $\left.\eta\right|_{D^{\prime}}: D^{\prime} \rightarrow B_{E}$ есть изоморфизм.

Сюръективность отображения $\eta: D^{\prime} \rightarrow B_{E}$. Мы должны показать, что любая коса из $B_{E}$ изотопна в $\Pi \times[0,1]$ mрехстраничной косе, т. е. трехстраничному плетению $T$, такому, что слово $w_{T}$ содержит только буквы $b_{i}$ и $d_{i}$.

Пусть $\mathscr{W}$ - плоскость в $\mathbb{R}^{3}$, параллельная линии переплета $l$ и разделяющая множества $E_{1}$ и $E_{2} \cup E_{3}$. Любой элемент из $B_{E_{1}}$ представим косой, не пересекающей плоскость $\mathscr{W}$. Это дает нам включение $B_{E_{1}} \hookrightarrow B_{E}$.

Для любого элемента $x \in B_{E} \subset H$ найдется натуральное $N$, такое, что элемент $\left(b_{2} d_{3}\right)^{N} x\left(b_{3} d_{2}\right)^{N}$ представляется словом, одновременно 2- и 3-сбалансированным, т.е.

$$
\left(b_{2} d_{3}\right)^{N} x\left(b_{3} d_{2}\right)^{N} \in B_{E_{1}} .
$$

Следовательно, достаточно показать, что $\eta\left(D^{\prime}\right) \supset B_{E_{1}}$.

Группа $B_{E_{1}}$ канонически изоморфна группе $B_{\mathbb{N}}$. Стандартные образующие $\sigma_{k}$ группы $B_{\mathbb{N}}$ реализуются в полугруппе $Y$ следующим образом:

$$
\sigma_{k}=d_{3}^{k-1} b_{2} d_{3} d_{2} b_{3} b_{3}^{k-1}=\eta\left(d_{3}^{k-1} u_{1} d_{3}^{1-k}\right), \quad k>0 .
$$

Мы видим, что $\sigma_{k} \in \eta\left(D^{\prime}\right)$; значит, $B_{E_{1}} \subset \eta\left(D^{\prime}\right)$.

Инъективность отображения $\eta: D^{\prime} \rightarrow B_{E}$. Ввиду (17) и (18) достаточно показать, что элементы

$$
\bar{\sigma}_{k}=d_{3}^{k-1} u_{1} d_{3}^{1-k}
$$

группы $D$ удовлетворяют соотношениям (15) и (16). Начнем с соотношения (16). Имеем

$$
\begin{aligned}
d_{3}^{2} u_{2} d_{3}^{-2} & =d_{3}^{2}\left(d_{3}^{-1} d_{1} d_{3} d_{1}^{-1}\right) d_{3}^{-2}=d_{3} d_{1} d_{3} d_{1}^{-1} d_{3}^{-2} \\
& =d_{2}^{-1} d_{3} d_{2} d_{3}^{-1}=u_{1} \\
\bar{\sigma}_{1} \bar{\sigma}_{2} \bar{\sigma}_{1}\left(\bar{\sigma}_{2} \bar{\sigma}_{1} \bar{\sigma}_{2}\right)^{-1} & =u_{1} d_{3} u_{1} d_{3}^{-1} u_{1} d_{3} u_{1}^{-1} d_{3}^{-1} u_{1}^{-1} d_{3} u_{1}^{-1} d_{3}^{-1} \\
& =u_{1} d_{3} u_{1} d_{3}^{-1}\left(d_{2}^{-1} d_{3} d_{2} d_{3}^{-1}\right) d_{3} u_{1}^{-1} d_{3}^{-1} u_{1}^{-1} d_{3} u_{1}^{-1} d_{3}^{-1} \\
& =u_{1} d_{3} u_{1} d_{1} d_{3} d_{2} u_{1}^{-1} d_{3}^{-1} u_{1}^{-1} d_{3} u_{1}^{-1} d_{3}^{-1} \\
& =u_{1} d_{3} u_{1} d_{1} d_{3} d_{2}\left(d_{3} d_{2}^{-1} d_{3}^{-1} d_{2}\right) d_{3}^{-1} u_{1}^{-1} d_{3} u_{1}^{-1} d_{3}^{-1} \\
& =u_{1} d_{3} u_{1} d_{1} d_{3} d_{1}^{-1} d_{2}^{-1} d_{3}^{-1} d_{2} d_{3}^{-1} u_{1}^{-1} d_{3} u_{1}^{-1} d_{3}^{-1} \\
& =u_{1} d_{3}\left(d_{2}^{-1} d_{3} d_{2} d_{3}^{-1}\right) d_{1} d_{3} d_{1}^{-1} d_{2}^{-1} d_{3}^{-1} d_{2} d_{3}^{-1} u_{1}^{-1} d_{3} u_{1}^{-1} d_{3}^{-1}
\end{aligned}
$$




$$
\begin{aligned}
& =u_{1} d_{3} d_{2}^{-1} d_{3} d_{2} u_{2} d_{2}^{-1} d_{3}^{-1} d_{2} d_{3}^{-1} u_{1}^{-1} d_{3} u_{1}^{-1} d_{3}^{-1} \\
& =u_{1} d_{3} d_{2}^{-1} d_{3} u_{2} d_{3}^{-1} d_{2} d_{3}^{-1} u_{1}^{-1} d_{3} u_{1}^{-1} d_{3}^{-1} \\
& =\left(d_{2}^{-1} d_{3} d_{2} d_{3}^{-1}\right) d_{3} d_{2}^{-1} d_{3} u_{2} d_{3}^{-1} d_{2} d_{3}^{-1} u_{1}^{-1} d_{3} u_{1}^{-1} d_{3}^{-1} \\
& =d_{2}^{-1} d_{3}^{2} u_{2} d_{3}^{-1} d_{2} d_{3}^{-1} u_{1}^{-1} d_{3} u_{1}^{-1} d_{3}^{-1} \\
& =d_{2}^{-1} d_{3}^{2} u_{2} d_{3}^{-1} d_{2} d_{3}^{-1}\left(d_{3} d_{2}^{-1} d_{3}^{-1} d_{2}\right) d_{3} u_{1}^{-1} d_{3}^{-1} \\
& =d_{2}^{-1} d_{3}^{2} u_{2} d_{3}^{-2} d_{2} d_{3} u_{1}^{-1} d_{3}^{-1}=d_{2}^{-1} u_{1} d_{2} d_{3} u_{1}^{-1} d_{3}^{-1} \\
& =d_{2}^{-1} u_{1} d_{1}^{-1} u_{1}^{-1} d_{3}^{-1}=d_{2}^{-1} d_{1}^{-1} d_{3}^{-1}=1 \\
\bar{\sigma}_{k} \bar{\sigma}_{k+1} \bar{\sigma}_{k}\left(\bar{\sigma}_{k+1} \bar{\sigma}_{k} \bar{\sigma}_{k+1}\right)^{-1} & =d_{3}^{k-1} \bar{\sigma}_{1} \bar{\sigma}_{2} \bar{\sigma}_{1}\left(\bar{\sigma}_{2} \bar{\sigma}_{1} \bar{\sigma}_{2}\right)^{-1} d_{3}^{1-k}=1 .
\end{aligned}
$$

Теперь индукцией по $k>0$ докажем, что в группе $D$ выполнены следующие соотношения:

$$
\begin{aligned}
d_{3}^{k} u_{1} d_{3}^{-k} & =d_{2}^{-k} u_{1} d_{2}^{k}, \\
u_{1}\left(d_{3}^{k+1} u_{1} d_{3}^{-k-1}\right) & =\left(d_{3}^{k+1} u_{1} d_{3}^{-k-1}\right) u_{1} .
\end{aligned}
$$

Для $k=1$ соотношение (21) следует из (12) и соотношения $d_{3}^{2} u_{2} d_{3}^{-2}=u_{1}$, доказанного выше. Действительно,

$$
u_{1}\left(d_{3}^{2} u_{1} d_{3}^{-2}\right)=d_{3}^{2} u_{2} u_{1} d_{3}^{-2}=d_{3}^{2} u_{1} u_{2} d_{3}^{-2}=\left(d_{3}^{2} u_{1} d_{3}^{-2}\right) u_{1} \text {. }
$$

Что касается $(20)$, то в случае $k=1$ мы имеем

$$
d_{3} u_{1} d_{3}^{-1}=d_{2}^{-1} d_{1}^{-1} u_{1} d_{1} d_{2}=d_{2}^{-1} u_{1} d_{2} \text {. }
$$

Предположим, что (20) и (21) выполнены для $0<k \leqslant n-1$. Тогда

$$
\begin{aligned}
d_{3}^{n} u_{1} d_{3}^{-n}=\left(u_{1} d_{3}\right)^{n-1} d_{3} u_{1} d_{3}^{-1}\left(u_{1} d_{3}\right)^{1-n} & =\left(d_{2}^{-1} d_{3} d_{2}\right)^{n-1} d_{2}^{-1} u_{1} d_{2}\left(d_{2}^{-1} d_{3} d_{2}\right)^{1-n} \\
& =d_{2}^{-1} d_{3}^{n-1} u_{1} d_{3}^{1-n} d_{2}=d_{2}^{-n} u_{1} d_{2}^{n}
\end{aligned}
$$

Первое из равенств получается применением соотношения (21) $n-1$ раз. Далее,

$$
\begin{aligned}
u_{1}\left(d_{3}^{n+1} u_{1} d_{3}^{-n-1}\right) & =u_{1} d_{3}^{2}\left(d_{2}^{1-n} u_{1} d_{2}^{n-1}\right) d_{3}^{-2}=d_{3}^{2} u_{2}\left(d_{2}^{1-n} u_{1} d_{2}^{n-1}\right) d_{3}^{-2} \\
& =d_{3}^{2}\left(d_{2}^{1-n} u_{1} d_{2}^{n-1}\right) u_{2} d_{3}^{-2}=d_{3}^{2}\left(d_{2}^{1-n} u_{1} d_{2}^{n-1}\right) d_{3}^{-2} u_{1} \\
& =\left(d_{3}^{n+1} u_{1} d_{3}^{-n-1}\right) u_{1}
\end{aligned}
$$

Из (21) мы имеем для $k>j+1$

$$
\bar{\sigma}_{j} \bar{\sigma}_{k}=d_{3}^{j-1} u_{1}\left(d_{3}^{k-j} u_{1} d_{3}^{j-k}\right) d_{3}^{1-j}=d_{3}^{j-1}\left(d_{3}^{k-j} u_{1} d_{3}^{j-k}\right) u_{1} d_{3}^{1-j}=\bar{\sigma}_{k} \bar{\sigma}_{j} .
$$

Это завершает доказательство пп. 1) и 2) теоремы 2.

Заметим, что из наших рассуждений следует, что все элементы $(19), k \in \mathbb{Z}$, удовлетворяют соотношениям (15) и (16). Эти элементы порождают подгруппу $D_{3} \cap D^{\prime}$. Таким образом, мы имеем вложение

$$
B_{E_{1} \cup E_{2}} \hookrightarrow D_{3} .
$$

В $D_{3}$ имеется также подгруппа, изоморфная $\mathbb{Z}$, которая порождена элементом $d_{3}$.

Любой элемент $x$ из $D_{3}$ имеет вид $x=y d_{3}^{k}$, где $y \in B_{E_{1} \cup E_{2}}$. Это означает, что группа $D_{3}$ изоморфна полупрямому произведению $D_{3} \cong B_{E_{1} \cup E_{2}} \lambda_{\xi} \mathbb{Z}$, где действие $\xi$ группы $\mathbb{Z}$ на $B_{E_{1} \cup E_{2}}$ задается формулой $\xi\left(d_{3}\right)\left(\bar{\sigma}_{k}\right)=\bar{\sigma}_{k-1}$. 
Нетрудно видеть, что подгруппа $\widehat{D}_{i}$ совпадает с $B_{E_{i}}, i \in \mathbb{Z}_{3}$. Мы оставляем доказательство п. 3) теоремы 2 читателю.

ЗАмЕЧАНИЕ 2. В явном виде изоморфизм $B_{\mathbb{Z}} \cong D^{\prime}$ можно записать следующим образом:

$$
\sigma_{k} \mapsto \begin{cases}d_{3}^{k-1} u_{1} d_{3}^{1-k}, & \text { если } k \geqslant 1, \\ d_{2}\left(d_{2}^{-1} d_{3}\right)^{k / 2} u_{1}\left(d_{2}^{-1} d_{3}\right)^{-k / 2} d_{2}^{-1}, & \text { если } k \leqslant 0, k \text { четно, } \\ \left(d_{2}^{-1} d_{3}\right)^{(k-1) / 2} u_{1}\left(d_{2}^{-1} d_{3}\right)^{(1-k) / 2}, & \text { если } k \leqslant-1, k \text { нечетно. }\end{cases}
$$

В завершение параграфа мы укажем одно алгебраическое свойство группы $D$, которое легко следует из геометрической реализации и является в определенном смысле характеристическим.

ПРЕДЛОЖЕНИЕ 1. Для яюбого $k>0, l \geqslant 0$ существует мономорфизм (очевидно, единственный) $\psi_{k, l}: D \rightarrow D$, такой, что

$$
\psi_{k, l}\left(d_{1}\right)=d_{2}^{l} d_{1}^{k} d_{2}^{-l}, \quad \psi_{k, l}\left(d_{2}\right)=d_{1}^{-l} d_{2}^{k} d_{1}^{l}
$$

Гомоморфизмь $\psi_{k, l}$ удовлетворяют соотношениям

$$
\psi_{k, l} \circ \psi_{k^{\prime}, l^{\prime}}=\psi_{k k^{\prime}, k l^{\prime}+l},
$$

которье, обратно, влекут за собой соотношения (10)-(12), задающие групny $D$.

\section{§2. Ленты-зацепления}

В этом параграфе мы применим нашу трехстраничную технику к лентамзацеплениям. Под лентой-зацеплением мы подразумеваем двумерное компактное PL-подмногообразие $R \subset \mathbb{R}^{3}$ (с границей), каждая компонента которого гомеоморфна либо кольцу $S^{1} \times[0,1]$, либо ленте Мёбиуса $S^{1} \ltimes[0,1]$. Понятие ленты-зацепления близко к понятию оснащенного зацепления. А именно, если разрешить только ориентируемые, т. е. гомеоморфные кольцу $S^{1} \times[0,1]$, компоненты, то изотопические классы таких лент-зацеплений будут находиться во взаимно однозначном соответствии с изотопическими классами оснащенных зацеплений. Для наших целей будет удобнее допускать и неориентируемые компоненты. Мы говорим, что ленты-зацепления $R_{1}, R_{2}$ эквивалентны, и пишем $R_{1} \sim R_{2}$, если они (объемлемо) изотопны в $\mathbb{R}^{3}$.

Имеется очевидный способ превращать трехстраничные диаграммы зацеплений в ленты-зацепления. Чтобы это сделать, нужно заменить каждое ребро на его малую окрестность в соответствующей полуплоскости $P_{i}$ (см. рис. 3 ). Лента-зацепление, полученное таким образом из трехстраничной диаграммы $L$, будет обозначаться через $\check{L}$.

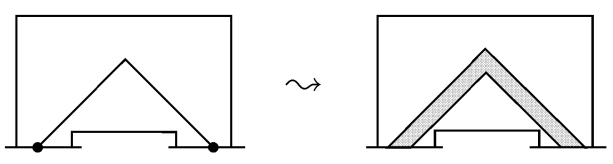

Рис. 3. Превращение трехстраничной диаграммы в ленту-зацепление 
Для лент-зацеплений мы построим полугруппу $\check{Y}$, аналогичную полугруппе $Y$ для обычных зацеплений. Полугруппа $\check{Y}$ порождается тем же множеством $\mathbb{A}$ и соотношениями

$$
\begin{gathered}
a_{i} d_{i}=a_{i+1} b_{i+1}, \quad b_{i} c_{i}=d_{i+1} c_{i+1}, \\
b_{i} b_{i-1} b_{i+1}=a_{i-1} c_{i+1} d_{i}, \quad d_{i} d_{i+1} d_{i-1}=a_{i+1} c_{i-1} b_{i}, \\
b_{i} d_{i}=d_{i} b_{i}=1, \\
a_{i} b_{i} c_{i}=b_{i}, \\
v w=w v,
\end{gathered}
$$

где $v \in\left\{a_{i-1} b_{i-1}, d_{i-1} c_{i-1}, b_{i+1} d_{i-1} d_{i+1} b_{i-1}, d_{i-1} d_{i} d_{i+1}\right\}, w \in\left\{a_{i}, b_{i}, c_{i}, b_{i-1} d_{i} d_{i-1}\right.$, $\left.d_{i+1} d_{i-1}\right\}, i \in \mathbb{Z}_{3}$. Для слова $w \in W$ мы будем обозначать задаваемый им элемент полугруппы $Y$ через $[w]$, а полугруппы $\check{Y}$ - через $\llbracket w \rrbracket$. Имеет место следующий аналог теоремы 1.

ТЕОРема 3. 1) Для произвольного ленть-зацепления $R$ существует трехстраничная диаграмма $L$, такая, что $R \sim \check{L}$;

2) для любых двух трехстраничных диаграмм $L_{1}, L_{2}$ ленты-зачепления $\check{L}_{1}$ и $\check{L}_{2}$ эквивалентнь тогда и только тогда, когда $\llbracket w_{L_{1}} \rrbracket=\llbracket w_{L_{2}} \rrbracket$;

3) элемент $x \in \check{Y}$ имеет вид $x=\llbracket w_{L} \rrbracket$, где $L-$ некоторая трехстраничная диаграмма, тогда и только тогда, когда он чентральный.

Геометрическая реализация полугруппы $\check{Y}$ почти такая же, как и полугруппы $Y$. Элементы полугруппы $\check{Y}$ могут быть реализованы как изотопические классы плетений, в которых все нити заменены на ленты. Доказательство последней теоремы отличается от доказательства теоремы 1 лишь в деталях, которые мы оставляем читателю.

Следующие два утверждения легко следуют из геометрической реализации полугрупп $Y$ и $\check{Y}$.

ПРЕДЛОЖЕНИЕ 2. Существует эпиморфизм $\theta: \check{Y} \rightarrow Y$, такой, что $\theta(\llbracket w \rrbracket)=$ $[w]$ для всех $w \in W$. Его ядро $\operatorname{ker}(\theta)$ изоморфно свободной абелевой группе $\mathbb{Z}_{\text {fin }}^{E}$, порожденной множеством $E$. Изоморфизм переводит $E_{i, k}$, где $i \in \mathbb{Z}_{3}, k \in \mathbb{N}$, в $\llbracket d_{i-1}^{k-1} b_{i+1} b_{i} b_{i-1}^{k} \rrbracket$.

ПРЕДЛОЖеНИЕ 3. Для любого $k>0$ существует гомоморфизм $\psi_{k}: \check{Y} \rightarrow \check{Y}$, maкой, чmo

$$
\psi_{k}\left(a_{i}\right)=a_{i}^{k}, \quad \psi_{k}\left(b_{i}\right)=b_{i}^{k}, \quad \psi_{k}\left(c_{i}\right)=c_{i}^{k}, \quad \psi_{k}\left(d_{i}\right)=d_{i}^{k}, \quad i \in \mathbb{Z}_{3} .
$$

Геометрически гомоморфизмы $\psi_{k}$ интерпретируются как локальное разрезание ленты на $k$ более узких лент (см. рис. 4).

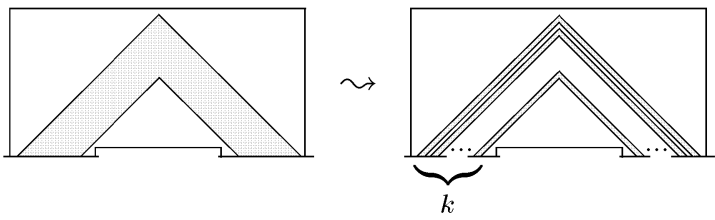

Рис. 4. Геометрическая реализация гомоморфизма $\psi_{k}$ 
Большинство известных инвариантов узлов и зацеплений учитывают ориентацию, в то время как наши конструкции хорошо приспособлены только к неориентированным (и нераскрашенным) зацеплениям. Поэтому связь между ориентированными инвариантами и полугруппами $Y$ и $\check{Y}$ еще предстоит выяснить. Однако существуют два многочлена Кауффмана, которые определены для неориентированных оснащенных зацеплений. Соответствующие теоремы легко переводятся на наш трехстраничный язык, что и сделано ниже.

Напомним, что скобочный многочлен Кауффмана [7] определяется скейнсоотношением

$$
\langle\otimes\rangle(A)-A \cdot\langle\rangle \bigotimes(A)-A^{-1} \cdot\langle\lesssim\rangle(A)=0
$$

и нормировкой

$$
\langle\bigcirc\rangle=1
$$

Скобочный многочлен удовлетворяет соотношению

$$
\langle\because\rangle(A)=-A^{3} \cdot\langle\|\rangle(A) \text {. }
$$

Мы можем продолжить его до инварианта лент-зацеплений, полагая

$$
\langle 0\rangle(A)=\sqrt{-A^{3}} \cdot\langle\|\rangle(A) \text {. }
$$

Пусть $\mathbb{Z}[\check{Y}]$ — полугрупповая алгебра полугруппы $\check{Y}, \mathbb{Z}[\check{Y}]\left[z, z^{-1}\right]$ - алгебра лорановских многочленов переменной $z$ с коэффициентами из $\mathbb{Z}[\check{Y}]$ и $K_{\langle\rangle}-$ факторалгебра

$$
\mathbb{Z}[\check{Y}]\left[z, z^{-1}\right] /\left(b_{3} b_{2} b_{1}-z^{3}, b_{3} b_{1}+d_{2} z+c_{1} a_{3} z^{2}\right) .
$$

Следующее утверждение есть трехстраничный вариант утверждения Кауффмана [7].

ПРЕДЛОЖЕНИЕ 4. 1) Канонический гомоморфизм $\mathbb{Z}\left[z, z^{-1}\right] \rightarrow K_{\langle\rangle}$инъективен.

2) Для яюбой трехстраничной диаграммь L имеет место следующее соотношение в алгебре $K_{\langle\rangle}$:

$$
\llbracket w_{L} \rrbracket=-\left(z^{4}+z^{-4}\right) \cdot\langle\check{L}\rangle\left(-z^{2}\right) .
$$

Аналогичная алгебра строится для L-многочлена $\operatorname{Kayффмана~} \mathcal{L}(z, \alpha)$, который определяется скейн-соотношениями

$$
\begin{aligned}
& \mathcal{L}_{\mathbb{S}}(z, \alpha)+\mathcal{L}(z, \alpha)=z \cdot\left(\mathcal{L}_{\mathbb{R}}(z, \alpha)+\mathcal{L}(z, \alpha)\right), \\
& \mathcal{L}_{\mathbb{0}}(z, \alpha)=\sqrt{\alpha} \cdot \mathcal{L}_{\rrbracket}(z, \alpha)
\end{aligned}
$$

и нормировкой

$$
\mathcal{L}_{\bigcirc}=1
$$

Пусть $K_{\mathcal{L}}$ - алгебра

$$
\mathbb{Z}[\check{Y}]\left[z, z^{-1}, t, t^{-1}\right] /\left(b_{3} b_{2} b_{1}-t, b_{3} b_{1} d_{3} d_{1}+b_{1} b_{3} d_{1} d_{3}-c_{1} a_{1} z-z\right) .
$$

Теорема Кауффмана [8, Theorem 2.3] о существовании и единственности L-многочлена записывается на трехстраничном языке следующим образом. 
ПРЕДЛОЖЕНИЕ 5. 1) Канонический гомоморфизм $\mathbb{Z}\left[z, z^{-1}, t, t^{-1}\right] \rightarrow K_{\mathcal{L}}$ инъективен.

2) Для яюбой трехстраничной диаграммь $L$ в алгебре $K_{\mathcal{L}}$ имеет место соотношение

$$
\llbracket w_{L} \rrbracket=\left(\left(t^{2}+t^{-2}\right) / z-1\right) \cdot \mathcal{L}_{\check{L}}\left(z, t^{2}\right) .
$$

В заключение я выражаю благодарность С. П. Новикову и В. Вершинину за высказанные критические замечания по этой работе, а также С. В. Матвееву за стимулирующее обсуждение на начальной стадии исследования.

\section{ЛИТЕРАТУРА}

1. Brunn H. Uber verknotete Kurven. Mathematiker-Kongresses Zurich, 1897, Leipzig, 1898, pp. 256-259.

2. Cromwell P. R. and Nutt I. J. Embedding knots and links in an open book. II: Bounds on arc index. Math. Proc. Camb. Philos. Soc., 119, No. 2, 309-319 (1996).

3. Morton H. R., Beltrami E. Arc index and the Kauffman poynomial. Math. Proc. Camb. Philos. Soc., 123, 41-48 (1998).

4. Дьнников И. А. Трехстраничное представление зацеплений. УМН, 53, вып. 5, 237-238 (1998).

5. Dynnikov I. A. A new way to represent links. One-dimensional formalism and untangling technology. Preprint, Moscow, 1998; http://mech.math.msu.su/ dynnikov.

6. Тураев В. Г. Операторные инварианты связок и $R$-матрицы. Изв. АН СССР, cep. матем., 53, № 5, 1073-1107 (1989).

7. Kauffman L. H. State models and the Jones polynomial. Topology, 26, No. 3, 395-407 (1987).

8. Kauffman L. H. An invariant of regular isotopy. Trans. Amer. Math. Soc., 318 (1990), no. 2, pp. 417-471.

9. Дьнников И. А. Трехстраничный подход в теории узлов. Кодирование и локальные движения. Функц. анализ и его прил., 33, вып. 4, 25-37 (1999).

Московский государственный университет, механико-математический факультет

Поступило в редакцию e-mail: dynnikov@mech.math.msu.su 